\title{
Síntesis y propiedades texturales de vidrios porosos de $\mathrm{SiO}_{2}$
}

\author{
Obdulia Medina*a ${ }^{\mathrm{a}}$ Fernando Rojas ${ }^{\mathrm{a}}$ \\ ${ }^{a}$ Departamento de Química, Universidad Autónoma Metropolitana-Iztapalapa, Av. San Rafael Atlixco 186, Col. Vicentina, CP 09340 \\ México, D.F. \\ *E-mail:mjyuyu@gmail.com
}

Recibido 08 Octubre 2013, Aceptado 10 Octubre 2013

\begin{abstract}
Resumen:
Existen cambios significativos en las propiedades texturalesde un vidrio precursor de $\mathrm{SiO}_{2}$ tanto por el efecto del tratamiento alternado acido-base, temperatura y por efecto de la desilización del substrato causada por tratamiento alcalino. Los tres tratamientos eliminan los microporos, concomitantemente aumenta el diámetro medio de poros y disminuye el área superficial, obteniéndose ciclos de histéresis IUPAC del Tipo HI en lugar de ciclos Tipo H2 propios del vidrio poroso, fenómeno que se refleja especialmente en distribuciones de tamaño de poro más homogéneas que la obtenida para el vidrio precursor. Esto último también se observa en las imágenes de MEB (SEM); el tratamiento térmico conduce a obtener curvas de adsorción-desorción de nitrógeno a 76Kque poseen una apreciable disminución del área superficial por efecto de sinterización. El efecto de desilización alcalina a concentraciones mayores a $0.30 \mathrm{M}$ de $\mathrm{NaOH}$ lleva a obtener ciclos de histéresis del tipo H4 en lugar del tipo H1. Finalmente, los parámetros experimentales idóneos que garantizan la obtención de vidrios porosos con áreas superficiales y volúmenes de poro eficientes para fines de una buena adsorción, incluyen: (i)los tratamientos alternados acido-base y (ii) el tratamiento térmico. Sin embargo, si existe interés en obtener materiales con mejor estabilidad mecánica, incluso a altas presiones, conviene desilizarlos vidrios precursores. Esta buena propiedad mecánica se atribuye a la presencia de las bandas vibracionales de los modosde flexión y estiramientotrasversales existentes para $\mathrm{SiO}_{2}$ a $800 \mathrm{~cm}^{-1}$ y $1080 \mathrm{~cm}^{-1}$ presentes en los espectros FTIR.
\end{abstract}

Palabras clave: sinterización, desilización, propiedades texturales, ciclos de histéresis.

\section{Introducción}

Los materiales porosos son afines a adsorbentes de interés y éstos a su vez se pueden diferenciar en términos del tipo de poro que presentan y se pueden clasificar al Modelo Dual de Sitios-Enlaces [1]. En particular, los vidrios de $\mathrm{SiO}_{2}$ son sólidos porosos interconectados a través de la matriz de sílice y forman parte de los sólidos conocidos como controlled pore glasses (CPG) [2].

La síntesis de vidrios porosos por el método sol-gel[3] es una alternativa eficiente, ya que controlando las condiciones de síntesis como temperatura[4], la relación TEOS (tertaetoxisilano) $/ \mathrm{H}_{2} \mathrm{O}$ y $\mathrm{pH}$ se pueden obtener vidrios con alta pureza y homogeneidad empleando temperaturas de síntesis relativamente bajas en comparación de la obtención de vidrios a través de la ruta cerámica tradicional. Esta alternativa de síntesis produce materiales porosos de $\mathrm{SiO}_{2}$ a partir de la solvatación de $\mathrm{SiO}_{4}$ tetraédrico que polimeriza hasta formar el gel como agregados de sílice que forman la estructura porosa con distintos tamaños de poro. Sin embargo, resulta más útil el obtener materiales porosos más homogéneos y con alta estabilidad mecánica para fines de buena adsorción.

Ciertos procesos de interés en adsorción requieren materiales porosos selectos en función del tamaño de poro [5], por ejemplo, para estudios de difusión generalmente se requieren materiales mesoporosos y existen materiales como las zeolitas que poseen microporos y para facilitar los fenómenos de trasporte generalmente se requiere de materiales mesoporosos. Por lo tanto el presente estudio explora los cambios en las propiedades texturales y morfológicas de un vidrio precursor de $\mathrm{SiO}_{2}$ por efectos, posteriores a la síntesis del mismo, de tratamientos acido-base[6], tratamientos térmicos y tratamiento por efecto de desilización [7].Se caracterizan los materiales vítreos resultantes por medio de diversas técnicas típicas de análisis instrumentales.

\section{Parte experimental}

Síntesis de Xerogeles de $\mathrm{SiO}_{2}$ : se obtuvo el vidrio poroso de sílice a partir de la hidrolisis y condensación de tetraetoxisilano (TEOS), de acuerdo a la relación molar [TEOS]: $\left[\mathrm{C}_{2} \mathrm{H}_{6} \mathrm{O}\right.$ (etanol)]: $\left[\mathrm{H}_{2} \mathrm{O}\right]$ 1:4:2.5.La reacción se llevó a cabo durante 2 días a $60^{\circ} \mathrm{C}$ en medio acido [8]. El vidrio resultante fue secado a $100^{\circ} \mathrm{C}$ y posteriormente calcinado a $500^{\circ} \mathrm{C}$ durante 4 días.

Para inferir los efectos de tratamientos posteriores a la síntesis del vidrio precursor de $\mathrm{SiO}_{2}$, se exploran los cambios ocasionados a las propiedades texturales por tratamiento en medio acido-base, en medio alcalino y por tratamiento térmico.

Tratamientos acido-base y térmicos: cada ciclo de lavado comprende tres etapas consecutivas: 1) tratamiento de $\mathrm{SiO}_{2}$ con la solución respectiva de $\mathrm{HCl}, 2$ ) hidrólisis de $\mathrm{SiO}_{2}$ y 3) tratamiento de $\mathrm{SiO}_{2}$ con la solución respectiva de $\mathrm{NaOH}$. En cada tratamiento de análisis prevalece la proporción, en masa 1:6 para $\mathrm{SiO}_{2}: \mathrm{H}_{2} \mathrm{O}$ y, asumiendo densidad de $1 \mathrm{~g} / \mathrm{cm}^{3}$ para $\mathrm{H}_{2} \mathrm{O}$, la misma porción en volumen de agua para cada medio de reacción (acido-base o puramente alcalino). El sistema se 
deja reaccionaren cada etapa del ciclo por un tiempo constante de 2 horas. Esto implica enjuagar la muestra de análisis después de cada separación del medio de reacción por filtrado. Los experimentos realizados se etiquetan de la siguiente manera:

Tabla1. Etiquetas de las muestras tratadas con $\mathrm{HCl}-\mathrm{NaOH}$ y sus correspondientes tratamientos térmicos.

\begin{tabular}{|c|c|c|c|c|c|c|c|c|}
\hline Tratamiento & A1 & $\mathrm{A2}$ & $\mathbf{A 3}$ & $\mathrm{A4}$ & A5 & $\overline{A 6}$ & A7 & A8 \\
\hline $\begin{array}{l}\mathrm{HCl}- \\
\mathrm{NaOH} 0.05 \mathrm{M}\end{array}$ & $5 \mathrm{Lv}$ & & $5 \mathrm{Lv}$ & & $10 \mathrm{Lv}$ & & $5 \mathrm{Lv}$ & \\
\hline $\begin{array}{l}\mathrm{HCl}- \\
\mathrm{NaOH} 0.10 \mathrm{M}\end{array}$ & & $5 \mathrm{Lv}$ & & $5 \mathrm{Lv}$ & & $10 \mathrm{~L}$ & & $5 \mathrm{Lv}$ \\
\hline $\begin{array}{l}\text { Secada } \\
65^{\circ} \mathrm{C}\end{array}$ & $3 \mathrm{D}$ & $3 \mathrm{D}$ & & & $3 \mathrm{D}$ & $3 \mathrm{D}$ & & \\
\hline $\begin{array}{l}\text { Calcinada a } \\
400^{\circ} \mathrm{C}\end{array}$ & & & 4D & $4 \mathrm{D}$ & & & 4D & $4 \mathrm{D}$ \\
\hline $\begin{array}{l}\text { Calcinada a } \\
450^{\circ} \mathrm{C}\end{array}$ & & & & & & & $4 \mathrm{D}$ & $4 \mathrm{D}$ \\
\hline $\begin{array}{l}\text { Las letras } \mathrm{L} \\
\text { D refiere a } \\
\text { calcinación } \\
\text { temperatura } \\
\text { temperature } \\
\text { enfriamient }\end{array}$ & dí & u & nún & ero de & $\begin{array}{l}\text { lavado } \\
\text { a mue }\end{array}$ & $\begin{array}{l}\text { reali } \\
\text { tra se }\end{array}$ & $\begin{array}{l}\text { ados, } \\
\text { ándo }\end{array}$ & $\begin{array}{l}\text { la letr } \\
\text { e o e } \\
\text { hpa d } \\
\text { lh, } 2\end{array}$ \\
\hline
\end{tabular}

Por ejemplo, la muestra A7 fue sometida a los siguientes procesos: 5 ciclos alternados de lavados con $\mathrm{HCl} 0.5 \mathrm{M}$ y $\mathrm{NaOH} 0.05 \mathrm{M}$, fue calcinada a $400^{\circ} \mathrm{C}$ durante 4 días y vuelta a calcinar durante otros 4 días a $450^{\circ} \mathrm{C}$.

Tratamientos alcalinos: en este caso cada ciclo de lavado comprende dos etapas consecutivas: 1) tratamiento de $\mathrm{SiO}_{2}$ con la solución a la concentración respectiva de $\mathrm{NaOH}, 2$ ) hidrólisis de $\mathrm{SiO}_{2}$. Esto se realiza manteniendo la proporción en masa 1:6 para $\mathrm{SiO}_{2}: \mathrm{H}_{2} \mathrm{O}$ y, asumiendo densidad de $1 \mathrm{~g} / \mathrm{cm}^{3}$ para $\mathrm{H}_{2} \mathrm{O}$, así como la misma porción en volumen de agua para cada medio de reacción alcalino. El sistema permanece a la temperatura deseada durante cada etapa del ciclo por un tiempo de 2 horas. Esto implica enjaguar la muestra de análisis después de cada separación del medio de reacción por filtrado y durante un tiempo de dos horas para cada ciclo de lavado. Las muestras obtenidas al final de completar 5 ciclos de lavados fueron secadas a $65^{\circ} \mathrm{C}$ durante 3 días y se les asignó la siguiente clasificación en función de, la única variable de análisis en estos experimentos (i.e. la concentración de NaOH): M1, M2, M3, M4, M5, M6, M7 y M8 para las concentraciones de $0.05 \mathrm{M}, 0.10 \mathrm{M}$, $0.15 \mathrm{M}, \quad 0.20 \mathrm{M}, \quad 0.25 \mathrm{M}, \quad 0.30 \mathrm{M}, \quad 0.35 \mathrm{M}$ у $0.40 \mathrm{M}$, respectivamente.

Caracterización de los vidrios de $\mathrm{SiO}_{2}$ : tanto para el precursor, como para todas las muestras de los distintos tratamientos, se realizaron experimentos de adsorción de $\mathrm{N}_{2}$ a $76 \mathrm{~K}$. Cada muestra fue desgasificada a $125^{\circ} \mathrm{C}$ durante $12 \mathrm{~h}$ en un equipo automático volumétrico Micromeritics ASAP 2020, Surface Area and Porosity Analyzer y se prosiguió con los análisis correspondientes a la de adsorción-desorción de N2.
Por otra parte, también se realizaron análisis de espectroscopia infrarroja de los adsorbentes empleando un espectrofotómetro Perkin Elmer FT-TR GX. Para el precursor y todas las muestras procedentes de los tratamientos acido-base o térmicos, se prepararon pastillas para su análisis sin $\mathrm{KBr}$. Sin embargo, para las muestras procedentes del tratamiento alcalino fue necesario emplear $\mathrm{KBr}$ previamente secado a $400^{\circ} \mathrm{C}$ por $12 \mathrm{~h}$ para obtener pastillas delgadas y trasparentes.

También se realizaron distribuciones de tamaño de poro por el método NLDFT [9] calculadas mediante el programa del equipo Autosorb $1 L$, modelado para sílice con un kernel para poros cilíndricos empleando la curva de desorción de $\mathrm{N}_{2}$ a $77 \mathrm{~K}$.

También se incluyen imágenes de SEM en el instrumento Jeol 7600F para las muestras del vidrio precursor y de los substratos resultantes de algunos de los tratamientos acido-base y térmicos correspondientes.

\section{Resultados y discusiones.}

La Tabla 2 muestra las propiedades texturales calculadas de las isotermas de sorción; es importante notar que el área de microporo $\left(\mathrm{A}_{\mathrm{MP}}\right)$ se calcula como:

$$
A_{M P}=A_{B E T}-A_{E X T}
$$

Donde $\mathrm{A}_{\mathrm{BET}}$ es el área BET superficial calculada en el intervalo de $\left.\mathrm{p} / \mathrm{p}^{0} 0.05,0.35\right], \mathrm{A}_{\mathrm{EXT}} \mathrm{se}$ refiere al área externa medida entre $\mathrm{p} / \mathrm{p}^{\circ} \quad[0.06$ a 0.60$]$. Por lo tanto, a medida que aumenta la intensidad del tratamiento químico, el área BET coincide cada vez más con él área externa, haciéndose evidente la disminución de área de microporos (ver Tabla 1).

Tabla 2. Propiedades texturales de los experimentos realizados sobre las muestras del vidrio precursor de $\mathrm{SiO}_{2}$; tratamientos ácido-base y térmicos.

\begin{tabular}{ccccc}
\hline Muestra: & $\begin{array}{c}\mathrm{A}_{\text {BET }} \\
\left(\mathrm{m}^{2} / \mathrm{g}\right)\end{array}$ & $\begin{array}{c}\mathrm{V}_{\text {DFT }} \\
\left(\mathrm{cm}^{3} / \mathrm{g}\right)\end{array}$ & $\begin{array}{c}\mathrm{A}_{\text {ext }} \\
\left(\mathrm{m}^{2} / \mathrm{g}\right)\end{array}$ & $\begin{array}{c}\mathrm{D}_{\text {NLDFT }} \\
\left(\mathrm{A}^{\circ}\right)\end{array}$ \\
\hline PRC: Precursor & 815.30 & 1.27 & 589.80 & 87 \\
$\mathrm{~A} 1: \mathrm{HCl}-\mathrm{NaOH}$ & 323.10 & 1.10 & 289.00 & 156 \\
$\begin{array}{c}0.05 \mathrm{M} \\
\mathrm{A} 2: \mathrm{HCl}-\mathrm{NaOH} \\
0.10 \mathrm{M}\end{array}$ & 285.70 & 1.18 & 272.50 & 177 \\
$\begin{array}{c}\mathrm{A} 3: \mathrm{A} 1 \mathrm{OM}+ \\
400^{\circ} \mathrm{C}\end{array}$ & 279.10 & 1.04 & 269.40 & 164 \\
$\begin{array}{c}\mathrm{A} 4: \mathrm{A} 2 \mathrm{OM}+ \\
400^{\circ} \mathrm{C}\end{array}$ & 235.60 & 1.11 & 214.70 & 195 \\
$\begin{array}{c}\mathrm{A} 5: 10 \mathrm{LvHCl}- \\
\mathrm{NaOH} 0.05 \mathrm{M}\end{array}$ & 273.50 & 1.12 & 242.70 & 183 \\
$\begin{array}{c}\mathrm{A} 6: 10 \mathrm{LvHCl}- \\
\mathrm{NaOH} 0.10 \mathrm{M}\end{array}$ & 235.80 & 1.06 & 217.50 & 192 \\
$\mathrm{~A} 7: \mathrm{A} 3 \mathrm{OM}+$ \\
$\begin{array}{c}450^{\circ} \mathrm{C} \\
\mathrm{A} 8: \mathrm{A} 4 \mathrm{OM}+ \\
450^{\circ} \mathrm{C}\end{array}$ & 253.30 & 1.10 & 245.70 & 180 \\
$\mathrm{M} 1 \mathrm{NaOH} 0.05 \mathrm{M}$ & 194.70 & 0.94 & 167.30 & 198 \\
$\mathrm{M} 2: \mathrm{NaOH} 0.10 \mathrm{M}$ & 107.10 & 0.52 & 88.46 & 199 \\
\hline
\end{tabular}




\begin{tabular}{lcccc}
\hline M3: NaOH 0.15M & 54.28 & 0.20 & 46.44 & 140 \\
M4: NaOH 0.20M & 14.95 & 0.06 & 11.84 & 141 \\
& & & & \\
M5: NaOH 0.25M & 7.06 & 0.02 & 5.23 & 118 \\
M6: NaOH 0.30M & 3.27 & 0.01 & 1.50 & 123 \\
M7: NaOH 0.35M & 9.50 & 0.03 & 7.20 & 139 \\
M8: NaOH 0.40M & 31.23 & 0.12 & 24.62 & 151 \\
\hline
\end{tabular}

Estas propiedades fueron determinadas para áreas: por el método $\operatorname{BET}\left(\mathrm{A}_{\mathrm{BET}}\right)$ a $\mathrm{p} / \mathrm{p}^{\circ}$ de $0.05-0.35$, método t para área externa $\left(\mathrm{A}_{\mathrm{ext}}\right.$ medida a 0.06-0.6 p/p $\mathrm{p}^{\circ}$. La distribución de tamaño de tamaño de poro se obtuvo por el Método NLDFT (para $\mathrm{N}_{2}$ a $77 \mathrm{~K}$ y para sílice considerando poros cilíndricos e isotermas de desorción).

Las isotermas de adsorción-desorción de nitrógeno a 76K para las muestras sometidas a tratamiento, acido-base, alcalino y tratamiento térmico, respecto a la muestra precursora de vidrio de $\mathrm{SiO}_{2}$ (Grafica 1 y Grafica 2), denotan una tendencia general hacia la disminución de área superficial. Sin embargo, se obtienen curvas más simétricas y características de sólidos porosos con tamaños más homogéneos para el precursor de sílice ciclos de histéresis $\mathrm{H} 2$, propios de vidrios porosos [10].En cambio para los substratos sometidos a los diversos tratamientos se observan ciclos de histéresis $\mathrm{H} 1$, a excepción de los tratamientos más intensos por efecto de desilización (a partir de la muestra M3 en adelante hasta M8).Estas muestras presentan ciclos de histéresis H4.Esto último hace evidente una pérdida de área superficial significativa por efecto de la lixiviación de $\mathrm{SiO}_{2}$ como se observa en la gráfica 2 .

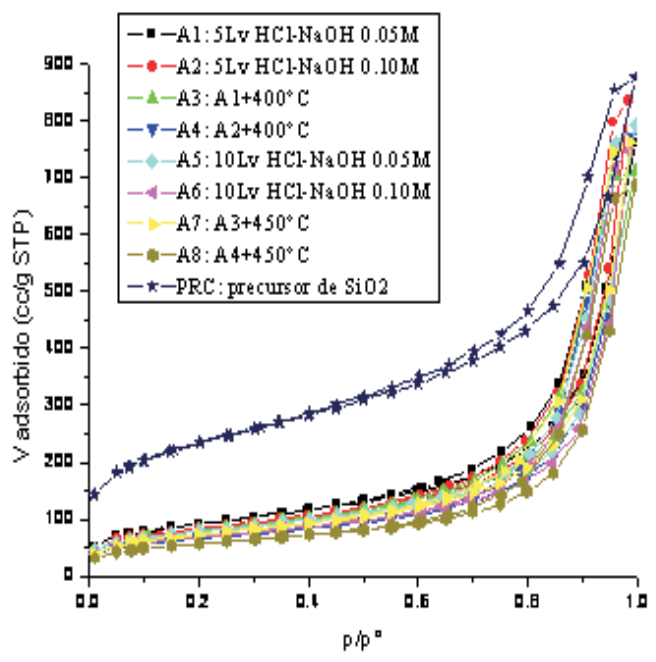

Gráfica 1. Isotermas de adsorción-desorciónde nitrógeno a 76K de las muestras procedentes del tratamiento acido-base y posterior tratamiento térmico.

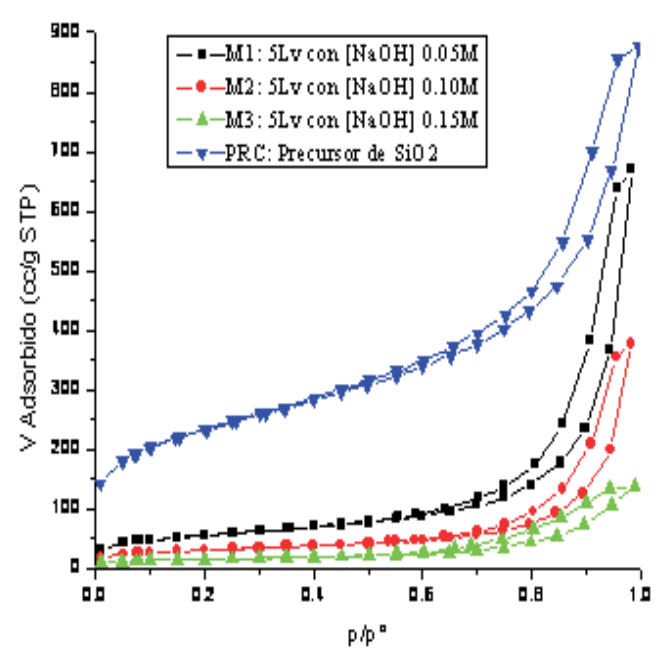

Gráfica 2. Isotermas de adsorción sobre las muestras resultantes del precursor sometido a tratamientos por con diferentes concentraciones de $\mathrm{NaOH}$.

Los cambios más significativos en perdida de área superficial, respecto al precursor, los sufrieron las muestras tratadas con $\mathrm{HCl}-\mathrm{NaOH} 0.1 \mathrm{M}$ por 10 ciclos (A6) con $63 \%$ de pérdida así como la muestra calcinada por segunda vez (A8) con $70 \%$. Sin embargo, con los experimentos menos agresivos de tratamiento alcalino (M1), se obtuvo una pérdida de área externa del $72 \%$, siendo así que al tratar al precursor de $\mathrm{SiO}_{2}$ solamente con soluciones de $\mathrm{NaOH}$, se observa un intenso proceso de lixiviación de sílice. En la gráfica 4, se observa esta aguda perdida de área externa la cual resulta ser asintótica.

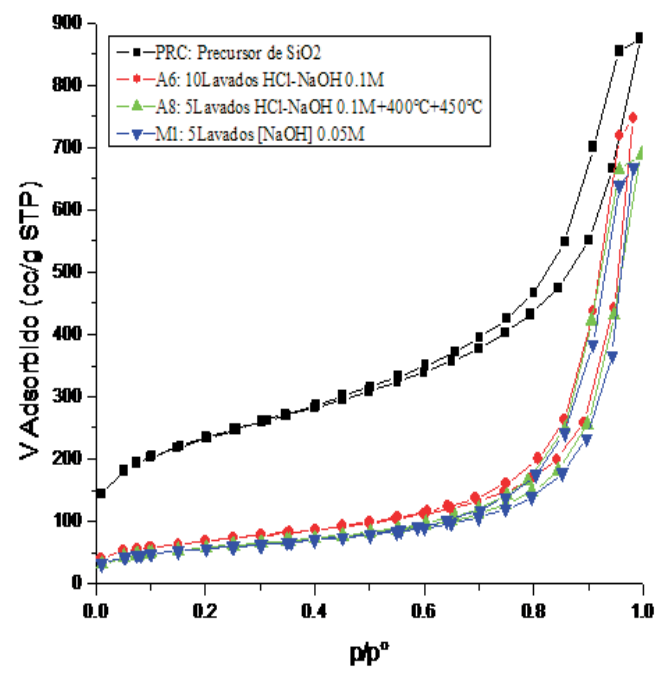

Gráfica 3. Isotermas de adsorción-desorción de nitrógeno a $76 \mathrm{~K}$ para los tratamientos más agresivos, después de cada prueba para el precursor de $\mathrm{SiO}_{2}$. 


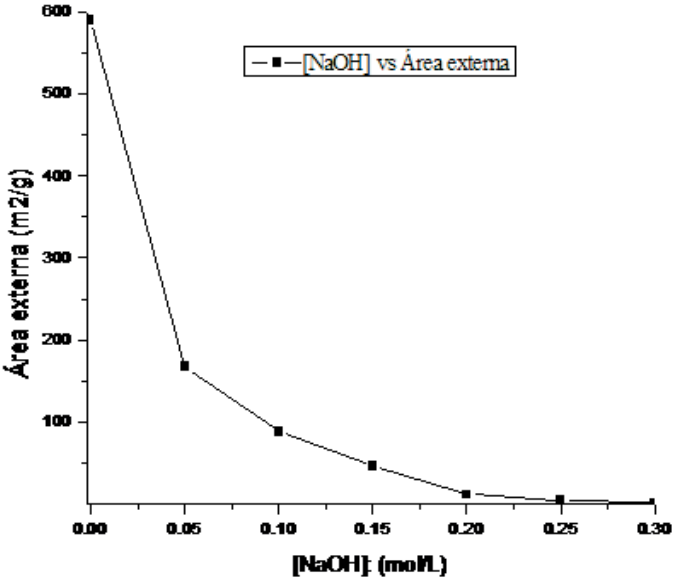

Gráfica4. Efecto de la pérdida de área superficial en función de la concentración de $\mathrm{NaOH}$.

En cuanto a las distribuciones de tamaño de poro determinadas por el método NLDFT se muestra (Graficas 5,6 y 7) que para el precursor de sílice existe una población de poros de diversos tamaños, con abundantes microporos. Para las muestras procedentes de los experimentos de ciclos de tratamiento acido-base y tratamiento por efecto de desilización alcalina, se tiene menor distribución de tamaños menos sinuosa (con respecto al precursor), sin embargo, al igual que para las curvas de adsorción se aprecia una ligera diferencia entre los tratamientos acido-base y térmicos, mientras para los tratamientos alcalinos es evidente el ataque que produce a la matriz de sílice.

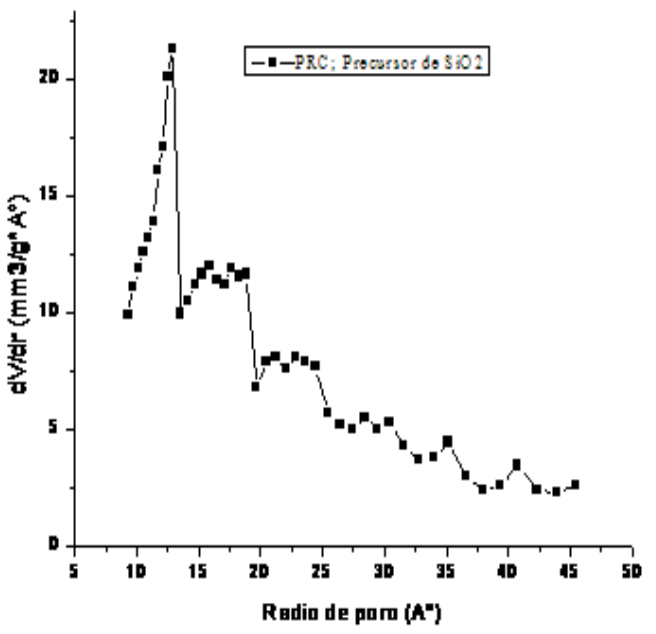

Gráfica 5. Distribución de tamaño de poro por el método NLDFT del vidrio precursor de $\mathrm{SiO}_{2}$.

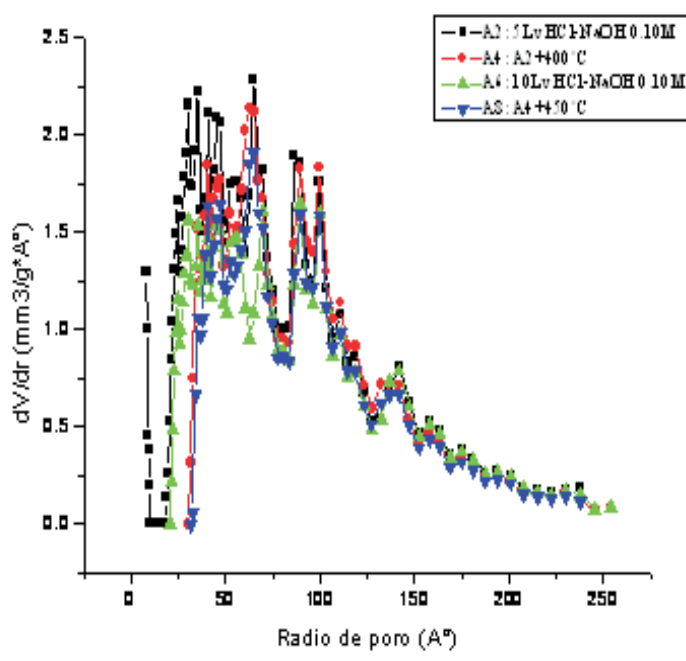

Gráfica6. Distribución de tamaño de poro por el método NLDFT para muestras de $\mathrm{SiO}_{2}$ tratadas con $\mathrm{HCl}-\mathrm{NaOH} 0.1 \mathrm{M}$.

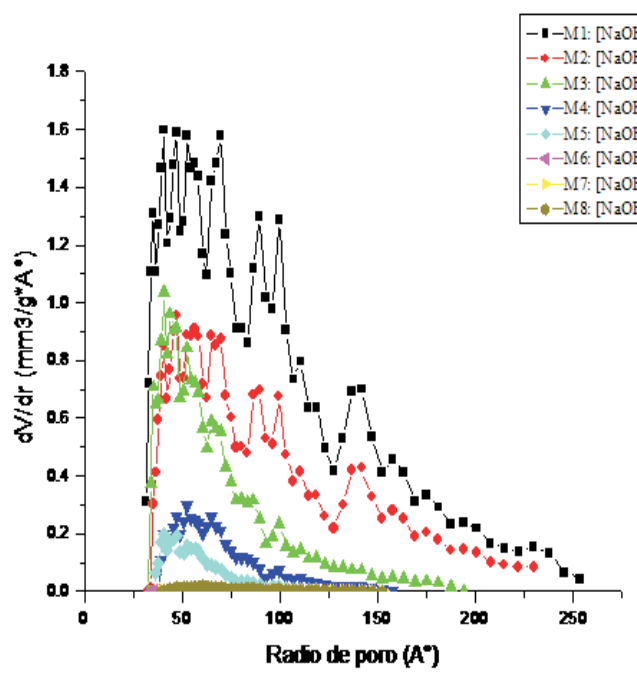

Gráfica7. Distribuciones de tamaño de poro por el método NLDFT para las muestras de precursor tratadas a diferentes concentraciones de $\mathrm{NaOH}$.

Referente a los espectros de infrarrojo (FTITR) para los vidrios de $\mathrm{SiO}_{2}$ [11-12] existen diferencias muy significativas en los modos vibracionales en términos de los diferentes tratamientos realizados. Sin embargo, el precursor de sílice muestra compatibilidad con los tratamientos acido-base ya que, en general, las señales más características aparecen en: $3750 \mathrm{~cm}^{-1}$ para los silanoles libres, $3690 \mathrm{~cm}^{-1}$ para $\mathrm{Si}-\mathrm{OH}$ aislados superficialmente, $\quad 1600 \mathrm{~cm}^{-1}$ correspondientes a deformación de agua contenida en $\mathrm{SiO}_{2}, 1400-1000 \mathrm{~cm}^{-1}$ : vibraciones de $\mathrm{CH}$ y $\mathrm{CH}_{2}$ y finalmente vibración de SiO-Si a $1130-1100 \mathrm{~cm}^{-1}$. Por otra parte, en lo que se refiere a los tratamientos por efecto de desilización alcalina se obtienen espectros que hacen evidentes las diferencias estructurales con los espectros previos, y en dónde las señales características son: nuevamente las 
bandas de agua adsorbida $\left(3400-3200 \mathrm{~cm}^{-1}\right)$ y las bandas de deformación de agua molecular fisisorbida $\left(1600 \mathrm{~cm}^{-1}\right)$ ;además ahora se observan las bandas a $950 \mathrm{~cm}^{-1}(810$ $-950 \mathrm{~cm}^{-1}$ ) asignadas a enlaces $\mathrm{Si}-\mathrm{OH}$ interaccionando vía puentes de hidrógeno y modos ópticos trasversales en $\mathrm{SiO}_{2}$ de balanceo, flexión y estiramiento 800 y 1080 $\mathrm{cm}^{-1}$, respectivamente. Cabe mencionar que los materiales tratados solamente con medio alcalino presentan propiedades mecánicas muy interesantes, ya que resultan ser vidrios añejos mucho más estables que los referentes al tratamiento acido-base y tratamiento térmico, propiedad atribuida a la presencia de las bandas vibracionales desacopladas. A pesar de que las muestras en medio alcalino no fueron calcinadas, éstas presentan señales menos intensas respecto a la presencia de agua dentro de la matriz de sílice comparadas con los experimentos con vidrios de $\mathrm{SiO}_{2}$ en medio acido-base o incluso para algunas muestras que se sometieron a tratamiento térmico.

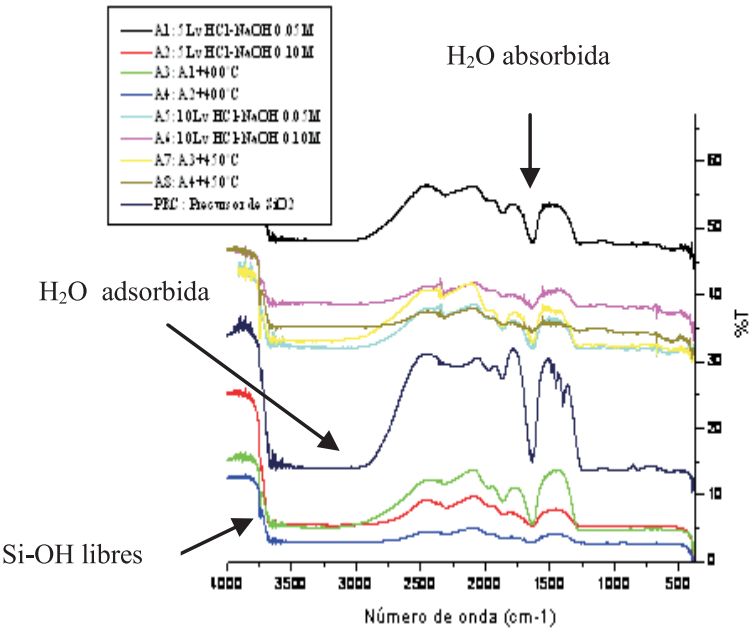

Gráfica8. Espectros infrarrojos del precursor, y de todas las muestras tratadas con soluciones ácido-base y de muestras sometidas a tratamiento térmico.

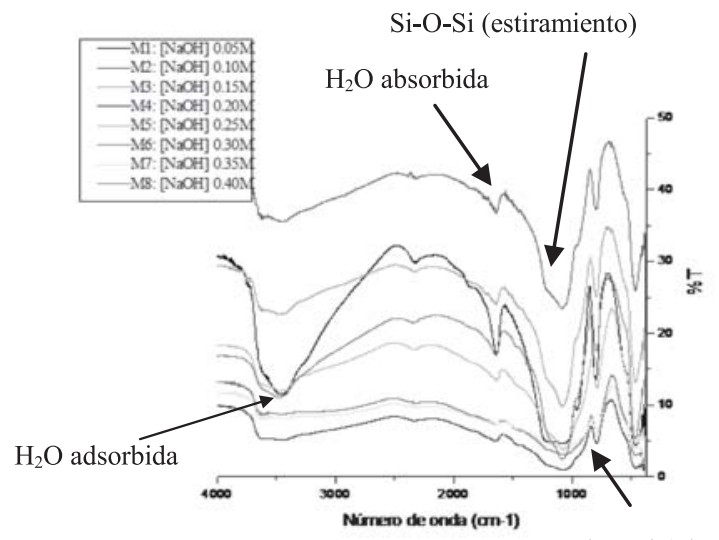

Si-O-Si (Flexión)

Gráfica9. Espectros infrarrojos de las muestras tratadas con solucionesalcalinas (usando $\mathrm{KBr}$ para hacer las pastillas); señales características de enlaces $\mathrm{O}-\mathrm{Si}-\mathrm{O}$ modos vibracionales desacoplados $\left(800\right.$ y $\left.1080 \mathrm{~cm}^{-1}\right)$.
Las imágenes de SEM (Imagénes 1-3) muestran la evolución del vidrio precursor en función de algunos de los tratamientos posteriores, siendo que para el precursor se aprecia una matriz de sílice más conglomerada y con poros heterogéneos (Imagen 1).Por otra parte para los tratamientos acido-base (Imagen 2) se obtiene una mayor porosidad aparente con poros granulares más ordenados al incrementar la cantidad de ciclos de lavados con $\mathrm{HCl}-$ $\mathrm{NaOH}$ 0.1M.Finalmente, para las muestras calcinadas, y previamente tratadas con 5 ciclos de lavados con $\mathrm{HCl}$ $\mathrm{NaOH}$ 0.1M (Imagen 3), se observa el fenómeno de sinterización de la matriz porosa de $\mathrm{SiO}_{2}$ por efecto de una segunda calcinación.

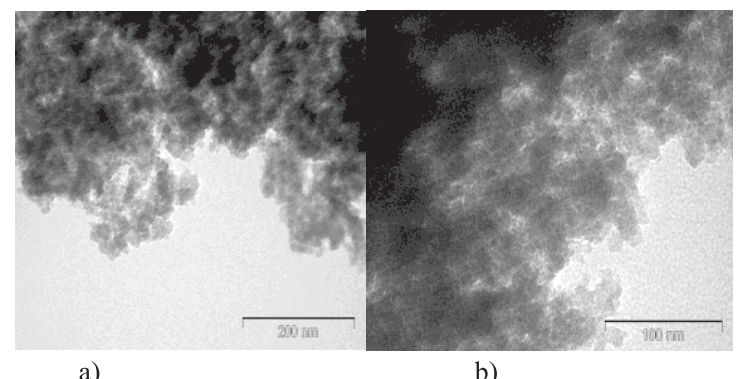

a)

b)

Imagen 1.a) Fotografía $\mathrm{SEM}$ de $\mathrm{SiO}_{2}$ precursor con resolución de $200 \mathrm{~nm}$ y b) Fotografía $\mathrm{SEM}$ de $\mathrm{SiO}_{2}$ precursor con resolución de $100 \mathrm{~nm}$.

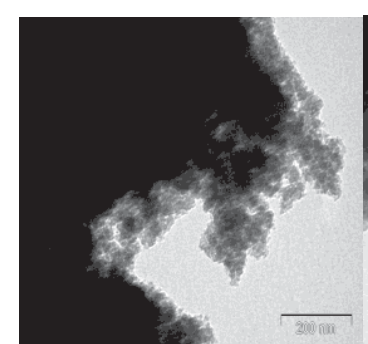

c)

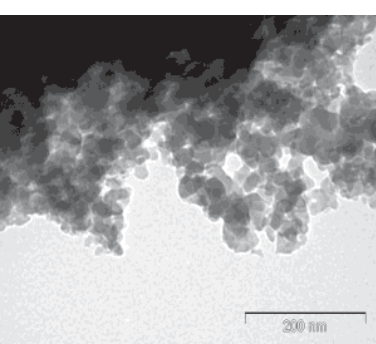

d)
Imagen 2.c) Fotografía SEM de precursor de sílice con 5 lavados con $\mathrm{HCl}-\mathrm{NaOH} 0.1 \mathrm{M}$ (muestra A2) y d) fotografía del precursor de sílice con 10 lavados con $\mathrm{HCl}-\mathrm{NaOH} \quad 0.1 \mathrm{M}$ (muestra A6).

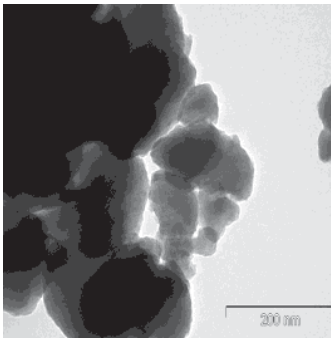

e)

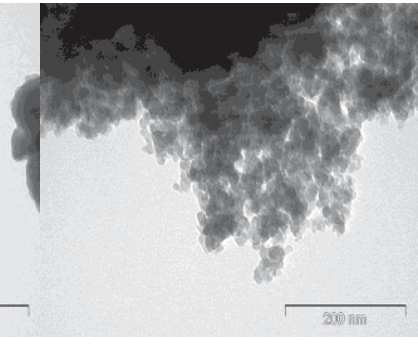

f)
Imagen 3. e) Fotografía SEM del precursor de sílice con 5 lavados en $\mathrm{HCl}-\mathrm{NaOH} 0.1 \mathrm{M}$ y calcinada a $400^{\circ} \mathrm{C}$ durante 4 días (muestra A4) y f) fotografía SEM del precursor de sílice con 5 lavados de $\mathrm{HCl}-\mathrm{NaOH} 0.1 \mathrm{M}$, calcinado a $400^{\circ} \mathrm{C}$ durante 4 días y con una segunda calcinación a $450^{\circ} \mathrm{C}$ por 4 días (muestra $\mathrm{A} 8$ ). 
Con la finalidad de resumir los efectos de pérdida de área externa sobre cada tratamiento, respecto al precursor de $\mathrm{SiO}_{2}$, se observa lo que se ha mostrado anteriormente (Tabla 2).Los efectos más agresivos relacionados con los tratamientos acido-base y térmico resultan ser comparables con aquellos derivados del tratamiento alcalino más suave al que fueron expuestas las muestras de $\mathrm{SiO}_{2}$ (observar Tabla 3).

Tabla 3. Resumen de pérdida de área externa (en \%) relativa respecto al área del precursor.

\begin{tabular}{|c|c|}
\hline Muestra: & $\%$ Perdida de $\mathrm{A}_{\text {ext }}$ \\
\hline PRC: Precursor & referencia \\
\hline A1: $\mathrm{HCl}-\mathrm{NaOH} 0.05 \mathrm{M}$ & 51 \\
\hline A2: $\mathrm{HCl}-\mathrm{NaOH} 0.10 \mathrm{M}$ & 54 \\
\hline $\mathrm{A} 3: \mathrm{A} 1 \mathrm{OM}+400^{\circ} \mathrm{C}$ & 54 \\
\hline $\mathrm{A} 4: \mathrm{A} 2 \mathrm{OM}+400^{\circ} \mathrm{C}$ & 64 \\
\hline A5: $10 \mathrm{LvHCl}-\mathrm{NaOH} 0.05 \mathrm{M}$ & 59 \\
\hline A6: $10 \mathrm{LvHCl}-\mathrm{NaOH} 0.10 \mathrm{M}$ & 63 \\
\hline $\mathrm{A} 7: \mathrm{A} 3 \mathrm{OM}+450^{\circ} \mathrm{C}$ & 58 \\
\hline $\mathrm{A} 8: \mathrm{A} 4 \mathrm{OM}+450^{\circ} \mathrm{C}$ & 70 \\
\hline M1: $\mathrm{NaOH} 0.05 \mathrm{M}$ & 72 \\
\hline M2: $\mathrm{NaOH} 0.10 \mathrm{M}$ & 85 \\
\hline M3: $\mathrm{NaOH} 0.15 \mathrm{M}$ & 92 \\
\hline $\mathrm{M} 4: \mathrm{NaOH} 0.20 \mathrm{M}$ & 98 \\
\hline M5: $\mathrm{NaOH} 0.25 \mathrm{M}$ & 99 \\
\hline M6: $\mathrm{NaOH} 0.30 \mathrm{M}$ & 100 \\
\hline M7: NaOH 0.35M & 99 \\
\hline M8: $\mathrm{NaOH} 0.40 \mathrm{M}$ & 96 \\
\hline
\end{tabular}

\section{Conclusiones}

Existe una tendencia general de pérdida de área superficial en función del medio de reacción (acido-base, o alcalino), a excepción de los tratamientos más agresivos con lavados con $\mathrm{NaOH}$ concentrada. En particular, se observó una pérdida de área superficial: A4< A8 < M1-M8poniendo en evidencia que el tratamiento por efecto de desilización es el más agresivo, ya que al incrementar la concentración de $\mathrm{NaOH}$ se observa una pérdida de área superficial externa drástica e incluso asintótica a cero (ver Grafica 4).

Todos los tratamientos eliminan parciamente los microporos, con diferentes cantidades, y concomitantemente aumentan el diámetro medio de poros y disminuyen el área superficial, obteniéndose ciclos de histéresis del Tipo HI en lugar de ciclos de histéresis Tipo $\mathrm{H} 2$ propios de los vidrios porosos de $\mathrm{SiO}_{2}$ precursores. Sin embargo, a medida que aumenta la concentración del $\mathrm{NaOH}$ los ciclos de histéresis pasan a ser del tipo $\mathrm{H} 4$ en vez de los tipos $\mathrm{H} 1$ y H2.
Existen poblaciones de señales distintas en los espectros FTIR para los vidrios $\mathrm{SiO}_{2}$ lavados con ácido-base, respecto a los tratados por efecto de desilización alcalina, respaldando así las diferencias estructurales encontradas. Mientras los tratamientos térmicos posteriores a la síntesis del precursor de $\mathrm{SiO}_{2}$ reducen el tamaño de los poros debido a la creación de una fase sinterizada; la hidrólisis en medio acido-base o puramente alcalino modifica el añejado siendo que las muestras A1-A8 (muestras con tratamiento alternado acido base) son menos resistentes a la ruptura de la estructura que las muestras M1-M8 (tratamiento por efecto de desilización alcalina), cuyas diferencias en propiedades de estabilidad mecánica del material se atribuye a la banda vibracional desacoplada de estiramiento de $\mathrm{SiO}_{2}(1080 \mathrm{~cm}-1)$ que es predominantemente intensa para las muestras de M1-M8, la cual no se observa en las muestras A1-A8.

\section{Agradecimientos}

Principalmente a Dr. Maximiliano Asomoza por contribuir colaborar con la muestra precursora de vidrio de $\mathrm{SiO}_{2}$ y por abrirnos las puertas de su laboratorio para realizar los experimentos realizados.

Gracias a CONACYT por la beca de maestría proporcionada a Obdulia Medina-Juaréz.

\section{Referencias}

1. Esparza, J.M.; Ojeda, M.L.; Campero A.; Hernández G.; Asomoza, M.; Cordero, S.; Kornhauser, I.; Rojas. F. J. Mol Cat A: Chem.2005, 228, 97-110.

2. Okonogi, S.; Oguchi, T.; Yonemochi, E.; Yamamoto. K. J.

Col Int Sci. 1999, 216-276.

3. Larry, L.; Jon, K. West.Chem.1990, 90.33-72 93.

4. J. Rouquerol, F. Rodríguez-Reinoso, K. S. W. Sing and K. K. Unger. Studies in Surface Science and Catalysis; 1 ed; Elsevier Science B. V. 1994, 87; pp 429-437.

5. Pérez-Ramíre, J.; Abelló, S.; Bonilla, A.; Johan, C. Adv. Fun. Mater. 2009, 19, 164 -172.

6. Cross and Haynes. Pore structure and properties of

Materials.1 ed. Vol. I. Part I. 1973, pp C-61, C-79.

7. Susarrey-Arce, A.; Hernández-Espinosa, M. A.; Rojas-

González, F.; Reed, C.; Petranovskii, V.; Licea, A.Part. Syst. Charact. 2010, 27, $100-111$.

8. Kawaguchi, T.; Hishikura, H.; Kokubu, J. J. Non-Cryst. Sol. $1984,63,61$

9. Ravikovitch, P. I.; Haller G. L.; Neimark, A. V. Adv.

Col. Int. Sci. 1998, 203, 76-77.

10. Sing, K. W.; Everett, D. H.; Haul, R. A.W.; Moscou, L.; Pierotti, R. A.; Rouquerol, J.; Siemieniewska, T. Pure \& Appl. Chem. 1985, 57, 603-619.

11. Martinez, J. R. Rev. Mex. Fis. 2002, 48(2), 142-149. 12. Gallardo, J., Galindo, P., Durán, A. Soc. Esp. Cerám. Vidr. 2001.40, 31-35. 